indicates that it is not possible to interchange different sets of atomic charges within existing force-fields and that the approach of the 'Force-field Consortium' (see this Column, November issue of Chimia $\mathbf{1 9 9 0 ,}$ $44,377)$, consisting in the de novo development of a completely new set of parameters, including atomic charges, is probably the best strategy towards a significant improvement of the performances of the models. Thanks to Schrödinger, the future of simulations based on Newtonian mechanics is indeed very promising, but it will require patient and careful selections of potential functions and parameter optimizations before enlarging the range of present applications to complex macromolecules and new materials.
Chimia 45 (1991) 92-93

(C) Schweiz. Chemiker-Verband; ISSN 0009-4293

\section{MoMo: a Molecular-Modelling Program}

\author{
Georg Gescheidt ${ }^{* u}$ ) and Elisabeth Novotny-Bregger ${ }^{b}$ )
}

MoMo, Version 6.1

Author: Max Dobler, Laboratorium für Organische Chemie, ETH-Zentrum, CH-8092 Zürich

Distributor: The program is distributed by the author, Tel.: 01/256 45 09, Bitnet: DOBLER@CZHETH5A

Fee: SFr. 20.-

MoMo runs on all Macintosh computers i.e. MacPlus, Mac SE, SE/30, Macintosh II, IIcx, IIci, IIfx. It prints to any dot or laser printer. For unknown reasons, the program may cause problems on Macintoshes equipped with a non-apple monitor.

Program size: 122 kByte.

RAM required: ca. 300-400 kByte.

Installation: no installation procedure; start MoMo from Disk or copy to harddisk and start.

Chemists involved in various fields of activity are now more and more frequently using computers when studying molecular structures. Therefore, some of the major scientific software houses offer packages for this purpose. MoMo, a program developed for in-house use by Prof. M. Dobler at the ETH in Zürich, shows us what an easily learnable and usable chemist's tool can look like.

\section{Program Description}

When you start the program MoMo, a typical Macintosh window equipped with a menu bar and some buttons on the left- and right-hand side of the screen opens (Fig.). Now, you can read up to 400 atoms into each of the five available windows. Cartesian or crystal coordinates are required to read in the molecules or molecular assemblies. Furthermore, it is possible to add up to 20 symmetry operations leading to a representation of crystal lattices.

\footnotetext{
*Correspondence : Dr. G. Gescheidt

a) Institut für Physikalische Chemie

Universität Basel

Klingelbergstrasse 80

$\mathrm{CH}-4056$ Basel

b) Laboratorium für organische Chemie

ETH-Zentrum

CH-8092 Zürich
}

After choosing a coordinate file of the adequate format, a model of the desired structure is displayed on the screen. By using the translation or rotation buttons of the MoMo window (see Fig.), the model is transformed step by step according to three orthogonal screen axes $x, y$, and $z$. After the selection of four atoms, the torsion angle is varied by pressing the 'torsion' button. The increments for the rotations and translations can be changed in four steps from 1 to $10^{\circ}$ and 0.02 to $0.5 \AA$.

When you click two nonbonded atoms, their distance will be displayed along with a dotted line between them. After pressing the 'geom' button on the left-hand side of the screen, it changes to 'ok' and gives you access to bond lengths, bond angles, and torsion angles if two, three, and four consecutively bonded atoms, respectively, are 'clicked'.
The 400 atoms read into one MoMo window may come from up to four molecules. Each of these can be made active and singly manipulated as described above. Docking procedures can be performed with up to 10 nonbonded distances selected, the changing distances being displayed simultaneously.

In addition, MoMo includes features to modify imported structures: $i$ ) A new atom is attached by selecting the atom to which it is bound; two further atoms have to be chosen in order to define the bond and dihedral angles. $i$ ) $\mathrm{H}$-atoms can be added, and iii) bonds, or molecular fragments are deleted by a simple select-and-cut procedure. Furthermore, it is possible to fuse molecules, to superpose molecules, and to align a molecule along a given vector.

There are four different graphic representations available: $i$ ) a stick-type representation, $i i)$ stick-type with bold bonds for atoms lying above the screen plane, iii) balland-stick, and $i v$ ) space-filling model. It is not possible to manipulate the models in the latter two drawing modes, i.e. they are static. In all drawing modes, the atom labels are highlighted as an option. A list of program features is summarized in the Table. MoMo does not include geometry-optimisation procedures.

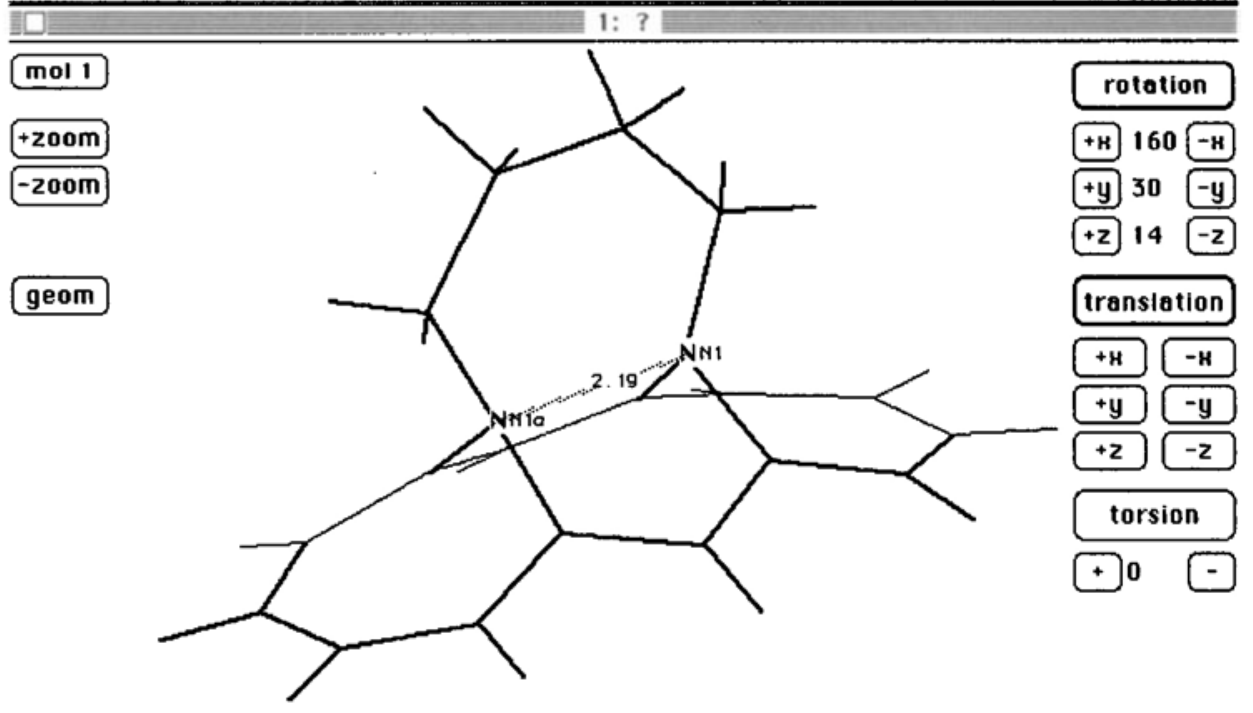


indicates that it is not possible to interchange different sets of atomic charges within existing force-fields and that the approach of the 'Force-field Consortium' (see this Column, November issue of Chimia $\mathbf{1 9 9 0 ,}$ $44,377)$, consisting in the de novo development of a completely new set of parameters, including atomic charges, is probably the best strategy towards a significant improvement of the performances of the models. Thanks to Schrödinger, the future of simulations based on Newtonian mechanics is indeed very promising, but it will require patient and careful selections of potential functions and parameter optimizations before enlarging the range of present applications to complex macromolecules and new materials.
Chimia 45 (1991) 92-93

(C) Schweiz. Chemiker-Verband; ISSN 0009-4293

\section{MoMo: a Molecular-Modelling Program}

\author{
Georg Gescheidt ${ }^{* u}$ ) and Elisabeth Novotny-Bregger ${ }^{b}$ )
}

MoMo, Version 6.1

Author: Max Dobler, Laboratorium für Organische Chemie, ETH-Zentrum, CH-8092 Zürich

Distributor: The program is distributed by the author, Tel.: 01/256 45 09, Bitnet: DOBLER@CZHETH5A

Fee: SFr. 20.-

MoMo runs on all Macintosh computers i.e. MacPlus, Mac SE, SE/30, Macintosh II, IIcx, IIci, IIfx. It prints to any dot or laser printer. For unknown reasons, the program may cause problems on Macintoshes equipped with a non-apple monitor.

Program size: 122 kByte.

RAM required: ca. 300-400 kByte.

Installation: no installation procedure; start MoMo from Disk or copy to harddisk and start.

Chemists involved in various fields of activity are now more and more frequently using computers when studying molecular structures. Therefore, some of the major scientific software houses offer packages for this purpose. MoMo, a program developed for in-house use by Prof. M. Dobler at the ETH in Zürich, shows us what an easily learnable and usable chemist's tool can look like.

\section{Program Description}

When you start the program MoMo, a typical Macintosh window equipped with a menu bar and some buttons on the left- and right-hand side of the screen opens (Fig.). Now, you can read up to 400 atoms into each of the five available windows. Cartesian or crystal coordinates are required to read in the molecules or molecular assemblies. Furthermore, it is possible to add up to 20 symmetry operations leading to a representation of crystal lattices.

\footnotetext{
*Correspondence : Dr. G. Gescheidt

a) Institut für Physikalische Chemie

Universität Basel

Klingelbergstrasse 80

$\mathrm{CH}-4056$ Basel

b) Laboratorium für organische Chemie

ETH-Zentrum

CH-8092 Zürich
}

After choosing a coordinate file of the adequate format, a model of the desired structure is displayed on the screen. By using the translation or rotation buttons of the MoMo window (see Fig.), the model is transformed step by step according to three orthogonal screen axes $x, y$, and $z$. After the selection of four atoms, the torsion angle is varied by pressing the 'torsion' button. The increments for the rotations and translations can be changed in four steps from 1 to $10^{\circ}$ and 0.02 to $0.5 \AA$.

When you click two nonbonded atoms, their distance will be displayed along with a dotted line between them. After pressing the 'geom' button on the left-hand side of the screen, it changes to 'ok' and gives you access to bond lengths, bond angles, and torsion angles if two, three, and four consecutively bonded atoms, respectively, are 'clicked'.
The 400 atoms read into one MoMo window may come from up to four molecules. Each of these can be made active and singly manipulated as described above. Docking procedures can be performed with up to 10 nonbonded distances selected, the changing distances being displayed simultaneously.

In addition, MoMo includes features to modify imported structures: $i$ ) A new atom is attached by selecting the atom to which it is bound; two further atoms have to be chosen in order to define the bond and dihedral angles. $i$ ) $\mathrm{H}$-atoms can be added, and iii) bonds, or molecular fragments are deleted by a simple select-and-cut procedure. Furthermore, it is possible to fuse molecules, to superpose molecules, and to align a molecule along a given vector.

There are four different graphic representations available: $i$ ) a stick-type representation, $i i)$ stick-type with bold bonds for atoms lying above the screen plane, iii) balland-stick, and $i v$ ) space-filling model. It is not possible to manipulate the models in the latter two drawing modes, i.e. they are static. In all drawing modes, the atom labels are highlighted as an option. A list of program features is summarized in the Table. MoMo does not include geometry-optimisation procedures.

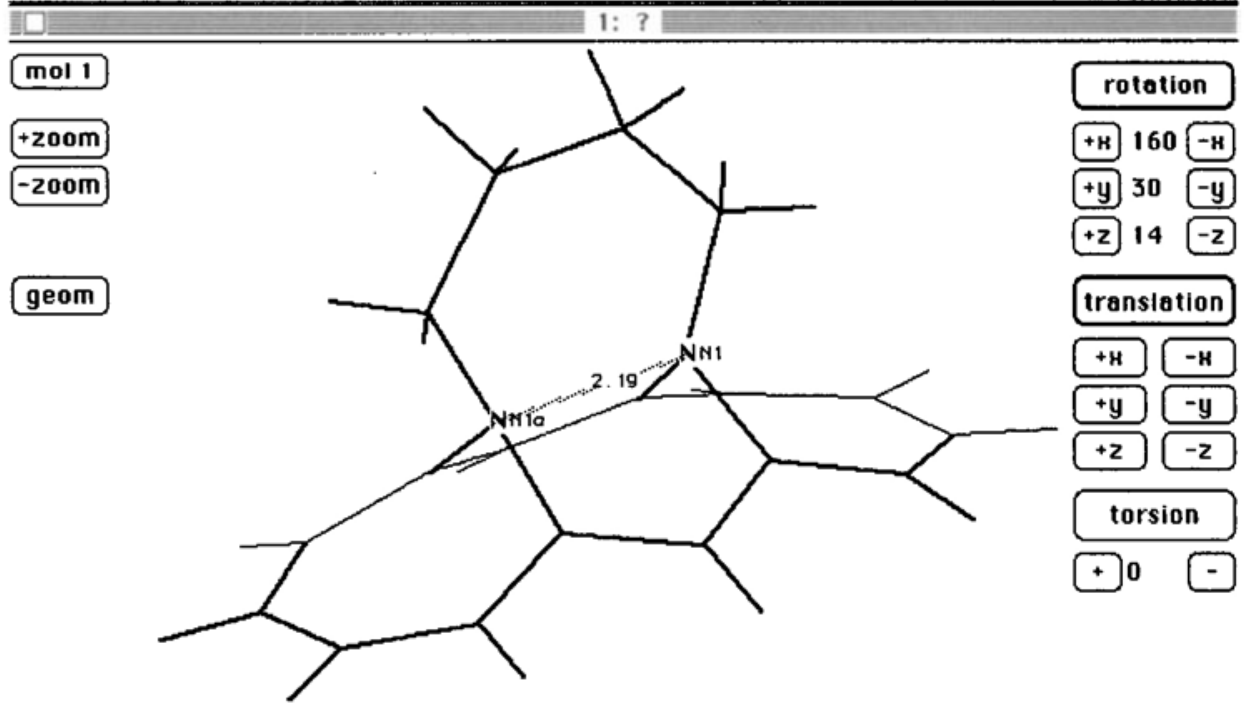




\section{Program Documentation}

With MoMo, you receive a short manual which sums up the features of the program. There is no tutorial for a non-experienced user. During program operation, the 'Help' pop-up menu offers on-line hints about some of the built-in features.

\section{Working with MoMo}

After reading in the coordinates of a molecule, the program displays a dialogue box which tells how many atoms and bonds are recognized. After this is confirmed ('ok' button), a stick-type representation of the structure is shown on the screen (Fig.). By using the rotation or translation buttons, the transformations proceed smoothly at nearly 'real-time', even if a high number of atoms are present. During the rotation procedure, the rotation angle for each of the axes is indicated between the corresponding buttons (Fig., upper right). With the help of these features, it is an easy task to view the three-dimensional arrangement of molecules at different orientations.

\section{In a Nutshell}

MoMo offers a convenient method to visualize models of structures with known coordinates. The interface is, typical for Macintosh computers, user friendly and easy to handle. The graphic representation is clear.

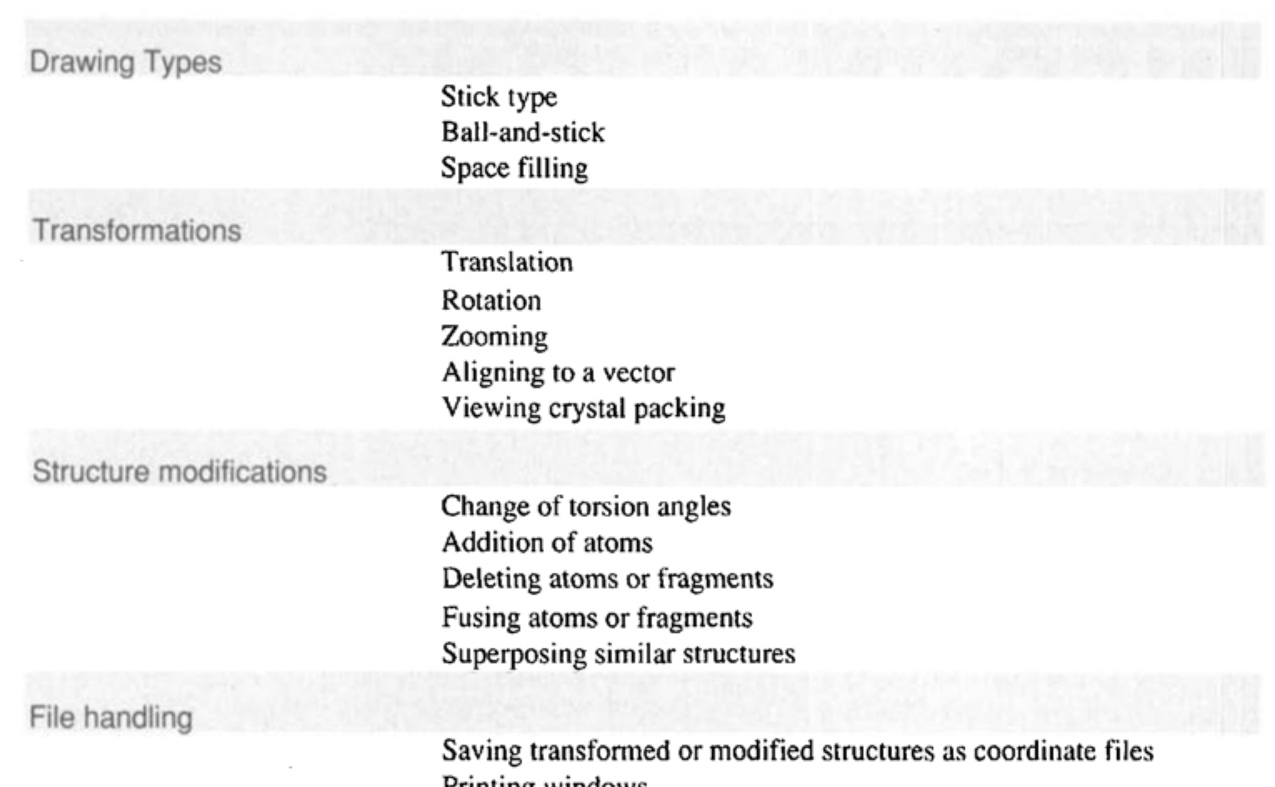

Printing windows

Table. Features of MoMo

After a rather short period of time, the user will be able to carry out a major part of the program facilities.

MoMo is not planned to be a tool to build up molecules, however, modification of an existing structure is an easy task. The files which the program accepts have a convenient format (Cartesian or crystal coordinates), so that coordinate files causing problems can be adjusted in any text editor.

There are some minor things which should be improved: $i$ ) if a file with a wrong format is read in, the program quits immediately (after giving an error message); it would be better, if there was a dialogue box which indicates that the file format is not correct and offers the possibility to choose a different file. ii) There is no possibility to export the graphics of the MoMo window to other applications using PICT or encapsulated postscript (EPS) files.

Whereas, in M.Ende's fairy tail 'Momo', you are asked to take your time, MoMo saves time and offers you a lot of molecularmodelling power on your Macintosh screen!

Received: December 21, 1990

\section{ANNOUNCEMENTS}

\section{Symposium für Theoretische Chemie}

Lage-Hörste bei Bielefeld, 1.-5. September 1991

Organisiert durch $J$. Hinze und H.-J. Werner (Fakultät für Chemie, Universität Bielefeld). Schwerpunkte auf den Gebieten «Elektronenkorrelation in Molekülen», «Berechnung und Theorie von Molekülspektren», «Dynamik chemischer Elementarprozesse» und «Nicht-adiabatische Effekte» durch Hauptvorträge von J. Almlöf, W. Domcke, J. Römelt, N. C. Handy, W. Kutzelnigg, P. Rosmus, K. Ruedenherg, J. Troe und L. Zülicke. Daneben sind Fachvorträge und Poster zu allen Themen der theoretischen Chemie erwünscht. Weitere Informationen erhalten Sie bei: Prof. H.-J. Werner, Fakultät für Chemie, Universität Bielefeld, D-4800 Bielefeld (EM: werner@chet01.unibielefeld.de).
Parallel Computing in Computational Chemistry and Physics

Vienna, Austria, September 16-27, 1991

Organized by Hans Lischka (Institute for Theoretical Chemistry, University of Vienna), in cooperation with Robert Harrison and Ron Shepard (Argonne National Laboratory), Hans Zima and Wolfgang Kleinert (Austrian Center for Parallel Computation), and Peter Rastl (Vienna University). This summer school consists of two parts: Part I teaches the basics of parallel computing, Part II is intended to be a workshop about the applications in chemistry and physics, where contributions from participants are welcome. For further information, contact: Prof. Hans Lischka, Institut für Theoretische Chemie, Universität Wien, Währingerstr. 17, A-1090 Wien (EM: Bitnet A8441DAA@AWIUNI11).
Workshop on Molecular Graphics: Basic Principles and Applications

Lausanne, October 1-2,1991

Organized by the Group of Swiss Computational Chemists. This workshop is intended to have a triple purpose: $i$ ) to present the basic principles and major tools of molecular graphics; $i i)$ to review some recent applications to chemistry, biochemistry, and drug design; iii) to enable the participants to have a first start in the practice of these techniques through guided exercises. Lectures by W.G. Richards (Oxford), J. Brickmann (Darmstadt), and H.P. Weber (Sandoz, Basel). A software vendor exhibit for commercial packages of molecular graphics will by organized. For further information, contact: Dr. P.-A. Carrupt, Institut de Chimie Therapeutique, Université de Lausanne, 3 place du Château, $\mathrm{CH}-1005$ Lausanne (Tel: 021/44 43 31; EM: CARRUPT@CLSUNI51). 
Schweizerischer Chemiker-Verband

Association Suisse des Chimistes

Swiss Association of Chemists

\section{Jahresbericht 1990 \\ des Schweizerischen Chemiker-Verbandes}

Das vergangene Jahr war wiederum stark geprägt durch die Kooperationstätigkeit des Schweizerischen Chemiker-Verbandes und der Schweizerischen Chemischen Gesellschaft. Der Bericht über die Tätigkeit des Koordinationsausschusses finden Sie in der Chimia 1990 , 44, 389.

Die bis jetzt erreichten Zwischenziele lassen sich wie folgt zusammenfassen: Gegenseitige Einsitznahme in den beiden Vorstanden, gemeinsame Abhaltung von Tagungen wie der Frühjahrs- und Herbstversammlungen, des Makromolekularen Symposiums und des Seminars 'Marketing, Forschung und Produktion'. Überdies wird die technische Redaktion der Chimia seit Nummer 4/90 von der Helvetica Chimica Acta Redaktion wahrgenommen.

Für die Vorbereitung des $\mathrm{Zu}$ sammenschlusses hat der Koordinationsausschuss, bestehend aus den Herren W. v. Philipshorn, W. Graf, G. Haas und A. Merbach, folgende Arbeitspapiere fertiggestellt: ein Organigramm über Struktur von Vorstand, Geschäftsleitung und Sektionen, Statuten für die neue Gesellschaft, ein 12-Punkte-Programm über neue Leistungen der neuen Gesellschaft und ein SektionsStatut. Darüber hinaus wurde ein Modellbudget für die neue Gesellschaft mit einem professionellen Sekretariat entwickelt.

Wie weiter? Im gegenwärtigen Zeitpunkt beschäftigt sich der Koordinationsausschuss mit der Erarbeitung von Vorschlägen zur personellen Besetzung der Vorstandsfunktionen in der neu zu gründenden Gesellschaft. Im weitern soll das ganze Geschäft bis zur Generalversammlung ' 91 soweit vorbereitet sein, dass die Mitglieder nach der positiv verlaufenen KonsultativAbstimmung von 1988 grundsätzlich über die Neugründung der Nachfolgegesellschaft befinden können. An einer a.o. Generalversammlung im Herbst 1991 soll dann der Beschluss gefasst werden, auf Datum der GV ' 92 den ChemikerVerband aufzulösen und in corpore der Nachfolgegesellschaft beizutreten. Der Präsident und der Vorstand bitten die Mitglieder, zahlreich an diesen zwei Versammlungen teilzunehmen. Damit bringen Sie zum Ausdruck, dass das Vorhaben 'Neugründung und Beitritt zu einer Nachfolgegesellschaft' auch Ihr Anliegen ist.

\section{Frühjahrstagung 1990}

Die Frühjahrstagung fand am 27. April 1990 bei der Lonza AG in Visp statt. Sie stand unter dem Titel: 'Integrierte Entsorgung im Werk Visp der Lonza $A G^{\prime}$. In vier Vorträgen wurde das Umweltschutz- und Entsorgungskonzept der Lonza, der Bau einer Reststoffdeponie, die Abfallentsorgung durch Verbrennung und das Konzept des produktionsintegrierten Umweltschutzes am Beispiel eines Verfahrens dargestellt. Diese Vorträge gaben einen hervorragenden Einblick in Konzepte und in den hohen Stand der Technik, de heute in der chemischen Produktion machbar ist. Eine Besichtigung vor Ort rundete darüber hinaus das Bild ab.

Diese vier Vorträge sind in der Chimia 1990, 44, 261 publizier worden.

\section{Generalversammlung}

Das Protokoll der 71. Generalversammlung ist in der Chimia 1990 44, 261 publiziert worden.

\section{Chimia}

Das abgelaufene Jahr war geprägt durch die Umstellung der Redaktion. Ab April ' 90 übernahm Prof. C. Ganter die wissenschaftliche Redaktionsverantwortung. Die technische Redaktion wird seit diesem Zeitpunkt von Dr. M.V. Kısakürek und von Frau C.Scheuss in der Redaktion der Helvetica Chimica Acta erledigt. Diese Umstellung brachte allen Beteiligten ein gehöriges Mass an Mehrarbeit. Ich möchte den betroffenen drei Personen dafür und für das Gelingen der Umstellung meinen besten-Dank aussprechen. Im vergangenen Jahr wurde auch die Zusammenarbeit $\mathrm{mi}$ Birkhäuser $+G B C$, Graphische Unternehmen, neu geregelt. Die Führungsverantwortung für die Chimia liegt ab 1.1.1991 vollständig in den Händen des Schweiz. ChemikerVerbandes. In seinem Auftrag ist die ASSA, Schweizer Annoncen AG, für die Inserateakquisition zuständig. Satz und Layout werden von der Firma Bruckmann \& Partner erledigt. Druck und Versand, sowie die administrativen Arbeiten für die Zeitschrift bleiben weiterhin be Birkhäuser $+G B C$ angesiedelt.

Im Jahre 1990 wurden in der Chimia 94 Artikel publiziert; nämlich 34 in der Rubrik Forschung, 30 in der Rubrik Technologie und 8 in der Columna Analytica bzw. Computational Chemistry. 10 Artike befassten sich mit Marketing Aspekten, 7 Artikel sind unter Diverse einzureihen und 5 Editorials wurden im abgelaufenen Jahr Publiziert. Letztere Kolumne wurde 1990 als neue Rubrik aufgenommen. Sie wird von den Herren Prof. $J$. Weber (Genf), PD Dr. H. Huber (Basel) und Dr. H.P. Weber (Basel) betreut, und erscheint im Wechsel mit derColumna Analytica von Prof M. Widmer. Der Gesamtumfang betrug 536 Seiten, wovon $355^{1 / 2}$ Seiten auf wissenschaftliche und technische Beiträge entfielen. $38 \frac{1}{2}$ Seiten beanspruchte der Informationsteil und 114 Seiten der Chimia Report mitsamt den Inseraten. Titelei und Jahresindex beliefen sich auf 28 Seiten

\section{Wissenschafiliche Symposien}

Am 20. und 21. September 1990 fand das '10th International Macromolecular Symposium' unter der Leitung von Dr. R. Darms in Interlaken statt. Unter dem Titel: 'Polymer Surfaces and Interfaces - Key to High Performance Materials präsentierten 12 Autoren hochstehende Beiträge zu diesem Gebiet. Die Referate wurden in der Chimia 1990, 44, 310-336 und 350-365 publiziert. Das Symposium wurde von 150 Teilnehmern besucht.

Folgende Veranstaltungen sind in nächster Zukunft geplant. 1991: 'I5th International Symposium on Column Liquid Chromatography', HPLC ' 91 vom 3.-7. Juni in Basel das 'Internationale Farbensymposium' vom 23.-26. September in Montreux. 1992: 'Symposium zum 100. Jahrestag der Genfer Nomenkalturkonferenz' vom 21./22. April in Genf, gleichzeitig findet die Gründungsversammlung der neuen Geselischaft statt; das '6th Seminar on Modern Synthetic Methods' von Prof. $R$. Scheffold in Interlaken. 1993: „Bio-organisches Symposium" unter der Leitung von Prof Kieslich, ebenfalls in Interlaken.

\section{Technische Weiterbildung}

Das Seminar 'Marketing-Forschung-Produktion' hat am 29./30.
März in Fribourg stattgefunden. Diese Art Veranstaltung für die technisch- bzw. produktionsorientierten Chemiker befriedigt ein echtes Bedürfnis, wie die ausnahmslos positiven Kommentare ersichtlich machen. Das Seminar wurde von 140 Teilnehmern besucht. Die Vorträge der 11 Referenten wurden in der Chimia 1990, 44, 123-155 publiziert.

\section{ILMAC}

Im Berichtsjahr fand die ILMAC ' 90 , die 11. Internationale ChemieFachmesse, vom 23.-26. Oktober in Basel statt. Sowohl die Fachmesse wie auch das parallel dazu stattfindende Basler Treffen für Chemische Technik waren ein vollerErfolg. Wie die Umfrage zeigte, waren sowohl Besucher wie Aussteller mit der Veranstaltung aufs Beste zufrieden. An der Eröffnung hielten Dr. $P h$. Lévy, Generaldirektor der Mustermesse, Dr. B.Glutz, Präsidentdes Organisations-Komitees, Begrüssungs- und Eröffnungsansprachen. Frau R. Simmen-Messmer, Ständerätin, schlug die Brücke zur Politik und Prof. W. Simon blickte in die Zukunft: Technologie und Einsatz von chemischen Sensoren/Biosensoren: Quo vadis? Im Rahmen der Eröffnungsveranstaltung wurde der ESCIS Chemie-Sicherheitspreis 1990 an Dr. Gerard Killé verliehen. Die Ansprachen und Referate des Eröffnungstages wurden in der Chimia 1990, 44, 390-398 publiziert.

\section{Mitglieder}

Am 31.12.1990verzeichnete der Verband 1039 Mitglieder (1989: 1053), nämlich 777 (778) ordentliche, 110(113) Senioren mit Chimia, 67 (68) Senioren ohne Chimia, 5 (5) Ehrenmitglieder, 26 (27) Studentenmitglieder und 54 (62) Firmenmitglieder. Insgesamt standen 27 (26) Neueintritten 29 (39) Austritte gegenüber. 12 (11) Mitglieder wurden wegen Nichtbezahlen des Mitgliederbeitrages ausgeschlossen.

\section{Auszeichnungen}

Im Berichtsjahr konnte sowohl der Preis des Schweiz. ChemikerVerbandes wie auch der Mar-LüthiPreis vergeben werden.

Der erste ging an Dr. Armin Reller, Universität Zürich, für seine Arbeiten über die Erzeugung organischer Verbindungen aus anorganischen Carbonaten mit Wasserstoff. Eine Publikation über diese Arbeiten wird in der Chimia 1991 erscheinen.

Der Max-Lüthi-Preis ging an Herm Marcel Sonderegger, Inge- 
nieurschule Winterthur, für seine Diplomarbeit: 'Kunststoff-Werkstoffe: Datenbanken, Verarbeitung, Charakterisierung'.

Dank

Dieses Jahr gilt mein besonderer Dank meinen Kollegen des Koordinationsausschusses. Die aktive und kompetente Mitarbeit der Herren W. v. Philipsborn, A. Merbach und $G$. Haas machten es möglich, dass wir bis Ende 1990 die entscheidenden Weichenstellungen zur Neugründung einer vereinigten Nachfolgegesellschaft für den Verband und die Gesellschaft machen konnten.

\section{Ausblick}

Am 18./19. April 1991 findet die 72. Generalversammlung und die Frühjahrstagung in Agno/Lugano im Tessin statt. Die Frühjahrstagung ist der Tessiner Pharmaindustrie gewidmet.

Im Herbst 1991 wird eine a.o. Generalversammlung, wahrscheinlich in Bern, stattfinden. Als einziges Traktandum wird die Auflösung des Chemiker-Verbandes und der Beitritt zur vereinigten Nachfolgegesellschaft zur Diskussion stehen.

\section{Walter Gra}

(Präsident)

Visp, Ende Februar 1991

\section{Schweizerische Chemische Gesellschaft} Sektion für Medizinische Chemie

Die Sektion für Medizinische Chemie (SMC) der Schweizerischen Chemischen Gesellschaft (SCG) wird am 22. Mai 1991 um 15.30 Uhr ihre zweite Mitgliederversammlung abhalten. Die Veranstaltung findet im Auditorium 510 bei Sandoz Pharma AG (Porte 5I, Hüningerstrasse 110, Basel) statt.

Auf dem Programm stehen u.a. eine Orientierung von Prof. W. von Philipshorn über den Stand und die Vorgeschichte des bevorstehenden Zusammenschlusses der SCG mit dem Schweizerischen Chemiker-

Verband (SChV), sowie zwei wissenschaftliche Vorträge von Prof. $H$. Timmermann (Amsterdam) 'Searching for selective ligands of histamine receptors'und Prof. Krogsgaard-Larsen (Kopenhagen) 'Drug Design Strategies in Alzheimer's Disease. Focus on Glutamic Acid, GABA and Acetylcholine'.

Interessenten sind herzlich eingeladen. Anmeldeformulare und weitere Auskünfte sind erhältlich beim SMC-Präsident Dr. E. Kyburz. c/o F. Hoffmann-La Roche $A G, 4002$ Basel.

\section{Neues Roche-Herz-Kreislauf-Medikament künftig gemeinsam von Roche und Marion Merrell Dow}

Marion Merrell Dow Inc. in Kansas City, Missouri/USA. und F.Hoffmann-La Roche AG in Basel, Schweiz. zusammen mit Hoffmann La-Roche Inc. In Nutley, New Jersey, haben soeben einen Vertrag unterzeichnet, der die gemeinsame Entwicklung und weltweite Vermarktung von Ro 40-5967, einem neuen Herzkreislauf-Medikament. vorsieht. Ro 40-5967 wurde in den Basler Laboratorien von Roche synthetisiert und stelt einen neuartigen Calciumantagonisten der dritten Generation dar: er befindet sich in Phase II der klinischen Entwicklung bei Bluthochdruck und Angina pectoris.

Fred W. Lyons, Jr., Präsident von Marion Merrell Dow Inc. kommentierte die Vereinbarung mit den Worten: „Wir meinen, dass unsere beiden Unternehmen sich ausgezeichnet in wissenschaftlicher Hinsicht wie auch im Marketing ergänzen und die notwendigen Ressourcen bereitstellen werden für die vollumfängliche Entwicklung und Vermarktung dieses vielversprechenden und für den Patienten äusserst wertvollen Medikaments. Wir erwarten eine lange und für beide Seiten lohnende Zusammenarbeit zwischen Marion Merrell Dow und Roche im Hinblick auf dieses Produkt."

Dr. Armin Kessler. Leiter der Pharma-Sparte und der konzernweiten operationellen Bereiche von F.Hoffmann-La Roche AG erklärte: „Normalerweise entwickeln und vermarkten grössere pharmazeutische Unternehmen ihre wichtigen, innovativen Substanzen selbst. Ro 40-5967 besitzt so interessante $\mathrm{Ei}$ genschaften. dass eine schnellstmögliche Entwicklung und Einführung am Markt erstrebenswert ist. Wir meinen, dass dies am besten durch die Kooperation unserer beiden Unternehmen erreicht werden kann, zumal sie sich in ihren Stärken vorteilhaft ergänzen."

Marion Merrell Dow vermarktet derzeit den Calciumantagonisten CARDIZEM (Diltiazem-HCl), ein gut eingeführtes Herz-KreislaufMedikament in den USA. Das Unternehmen bringt deshalb ein hohes Mass an Erfahrung in die gemeinsame Entwicklung und Vermarktung von Ro 40-5967 ein.
Schweizerischer Chemiker-Verband

Association Suisse des Chimistes

Schweizerische Chemische Gesellschaft

\section{Sehr geehrte Damen und Herren}

Wir freuen uns, unsere Mitglieder und Gäste zur Frühjahrstagung und zur ordentlichen Generalversammlung 1991 einzuladen.

Die Frühjahrstagung findet statt am

Donnerstag abend/Freitag, 18. und 19. April 1991

um 18.30 Uhr bzw. 08.30 Uhr, Hotel La Perla in Agno

The'ma der Tagung:

Aspekte der pharmazeutischen und chemischen Industrie im Tessin

Die Vorträge finden statt am Donnerstag, 18. April von 18.30-20.00 Uhr, Hotel La Perla, Agno. Besichtigt werden am Freitag, 19. April von 08.30-12.00 Uhr die Firmen Inpharzam, Pharmaton und Sapec. Die Firma Helsinn kann nach der GV am Freitag abend besichtigt werden.

Die Generalversammlung 1991 findet statt am

Freitag um 14.15 Uhr, Hotel La Perla, Agno

Die Frühjahrstagung wird gesponsort von den Firmen Helsinn SA, Biasca, Inpharzam SA, Cadempino und Taverne, Pharmaton SA, Bioggio und Sapec SA, Barbengo und von der Associazione Ticinese Industrie Chimiche e Farmaceutiche (ATICEF)

\section{Programm}

\section{Donnerstag 18. April 1991}

18.30 Begrüssung durch Dr. Walter Graf

Präsident des Schweiz. Chemiker-Verbandes Begrüssung durch Dr. Francesco Brigatti Präsident der Associazione Ticinese Industrie Chimiche e Farmaceutiche (ATICEF)

Begrüssung durch Dr. F. Giovannini

Vertreter des Organisationskomitees

18.45 Vorstellung der Firma Helsinn SA, Biasca, durch Dr. Rolf Wandeler, Geschäftsleiter Helsinn Biasc

19.05 Vorstellung der Firma Inpharzam SA, Cadempino, durch Dr. Anibale Gazzaniga Geschäftsleiter Inpharzam Ricerche Taverne

19.25 Vorstellung der Firma Pharmaton SA, Bioggio. durch Dr. Costante Mombelli stv. Generaldirektor Pharmaton Bioggio

19.45 Vorstellung der Firma Sapec SA, Lugano, durch Dr. Attilio Melera, Geschäftsleiter Sapec Barbengo

20.00 Cocktail und gemeinsames Nachtessen im Hotel La Perla in gemütlichem Rahmen.

Übernachten im Hotel La Perla, Agno

Freitag, 19. April 1991

8.30- Besichtigung in Gruppen der Firmen Inpharzam SA

12.00 Pharmaton SA, Sapec SA

12.30 Mittagessen

14.15 72. Generalversammlung des Schweizerischen ChemikerVerbandes im Hotel La Perla, Agno

ca. 16.00 Ende der Versammlung

ca. 17.00 Besichtigung der Firma Helsinn SA, Biasca (Autobahn-Anschluss Biasca, Richtung Industrie-Quartier)

Die genaue Zeit wird am Ende der GV bekannt gegeben. 
nieurschule Winterthur, für seine Diplomarbeit: 'Kunststoff-Werkstoffe: Datenbanken, Verarbeitung, Charakterisierung'.

Dank

Dieses Jahr gilt mein besonderer Dank meinen Kollegen des Koordinationsausschusses. Die aktive und kompetente Mitarbeit der Herren W. v. Philipsborn, A. Merbach und $G$. Haas machten es möglich, dass wir bis Ende 1990 die entscheidenden Weichenstellungen zur Neugründung einer vereinigten Nachfolgegesellschaft für den Verband und die Gesellschaft machen konnten.

\section{Ausblick}

Am 18./19. April 1991 findet die 72. Generalversammlung und die Frühjahrstagung in Agno/Lugano im Tessin statt. Die Frühjahrstagung ist der Tessiner Pharmaindustrie gewidmet.

Im Herbst 1991 wird eine a.o. Generalversammlung, wahrscheinlich in Bern, stattfinden. Als einziges Traktandum wird die Auflösung des Chemiker-Verbandes und der Beitritt zur vereinigten Nachfolgegesellschaft zur Diskussion stehen.

\section{Walter Gra}

(Präsident)

Visp, Ende Februar 1991

\section{Schweizerische Chemische Gesellschaft} Sektion für Medizinische Chemie

Die Sektion für Medizinische Chemie (SMC) der Schweizerischen Chemischen Gesellschaft (SCG) wird am 22. Mai 1991 um 15.30 Uhr ihre zweite Mitgliederversammlung abhalten. Die Veranstaltung findet im Auditorium 510 bei Sandoz Pharma AG (Porte 5I, Hüningerstrasse 110, Basel) statt.

Auf dem Programm stehen u.a. eine Orientierung von Prof. W. von Philipshorn über den Stand und die Vorgeschichte des bevorstehenden Zusammenschlusses der SCG mit dem Schweizerischen Chemiker-

Verband (SChV), sowie zwei wissenschaftliche Vorträge von Prof. $H$. Timmermann (Amsterdam) 'Searching for selective ligands of histamine receptors'und Prof. Krogsgaard-Larsen (Kopenhagen) 'Drug Design Strategies in Alzheimer's Disease. Focus on Glutamic Acid, GABA and Acetylcholine'.

Interessenten sind herzlich eingeladen. Anmeldeformulare und weitere Auskünfte sind erhältlich beim SMC-Präsident Dr. E. Kyburz. c/o F. Hoffmann-La Roche $A G, 4002$ Basel.

\section{Neues Roche-Herz-Kreislauf-Medikament künftig gemeinsam von Roche und Marion Merrell Dow}

Marion Merrell Dow Inc. in Kansas City, Missouri/USA. und F.Hoffmann-La Roche AG in Basel, Schweiz. zusammen mit Hoffmann La-Roche Inc. In Nutley, New Jersey, haben soeben einen Vertrag unterzeichnet, der die gemeinsame Entwicklung und weltweite Vermarktung von Ro 40-5967, einem neuen Herzkreislauf-Medikament. vorsieht. Ro 40-5967 wurde in den Basler Laboratorien von Roche synthetisiert und stelt einen neuartigen Calciumantagonisten der dritten Generation dar: er befindet sich in Phase II der klinischen Entwicklung bei Bluthochdruck und Angina pectoris.

Fred W. Lyons, Jr., Präsident von Marion Merrell Dow Inc. kommentierte die Vereinbarung mit den Worten: „Wir meinen, dass unsere beiden Unternehmen sich ausgezeichnet in wissenschaftlicher Hinsicht wie auch im Marketing ergänzen und die notwendigen Ressourcen bereitstellen werden für die vollumfängliche Entwicklung und Vermarktung dieses vielversprechenden und für den Patienten äusserst wertvollen Medikaments. Wir erwarten eine lange und für beide Seiten lohnende Zusammenarbeit zwischen Marion Merrell Dow und Roche im Hinblick auf dieses Produkt."

Dr. Armin Kessler. Leiter der Pharma-Sparte und der konzernweiten operationellen Bereiche von F.Hoffmann-La Roche AG erklärte: „Normalerweise entwickeln und vermarkten grössere pharmazeutische Unternehmen ihre wichtigen, innovativen Substanzen selbst. Ro 40-5967 besitzt so interessante $\mathrm{Ei}$ genschaften. dass eine schnellstmögliche Entwicklung und Einführung am Markt erstrebenswert ist. Wir meinen, dass dies am besten durch die Kooperation unserer beiden Unternehmen erreicht werden kann, zumal sie sich in ihren Stärken vorteilhaft ergänzen."

Marion Merrell Dow vermarktet derzeit den Calciumantagonisten CARDIZEM (Diltiazem-HCl), ein gut eingeführtes Herz-KreislaufMedikament in den USA. Das Unternehmen bringt deshalb ein hohes Mass an Erfahrung in die gemeinsame Entwicklung und Vermarktung von Ro 40-5967 ein.
Schweizerischer Chemiker-Verband

Association Suisse des Chimistes

Schweizerische Chemische Gesellschaft

\section{Sehr geehrte Damen und Herren}

Wir freuen uns, unsere Mitglieder und Gäste zur Frühjahrstagung und zur ordentlichen Generalversammlung 1991 einzuladen.

Die Frühjahrstagung findet statt am

Donnerstag abend/Freitag, 18. und 19. April 1991

um 18.30 Uhr bzw. 08.30 Uhr, Hotel La Perla in Agno

The'ma der Tagung:

Aspekte der pharmazeutischen und chemischen Industrie im Tessin

Die Vorträge finden statt am Donnerstag, 18. April von 18.30-20.00 Uhr, Hotel La Perla, Agno. Besichtigt werden am Freitag, 19. April von 08.30-12.00 Uhr die Firmen Inpharzam, Pharmaton und Sapec. Die Firma Helsinn kann nach der GV am Freitag abend besichtigt werden.

Die Generalversammlung 1991 findet statt am

Freitag um 14.15 Uhr, Hotel La Perla, Agno

Die Frühjahrstagung wird gesponsort von den Firmen Helsinn SA, Biasca, Inpharzam SA, Cadempino und Taverne, Pharmaton SA, Bioggio und Sapec SA, Barbengo und von der Associazione Ticinese Industrie Chimiche e Farmaceutiche (ATICEF)

\section{Programm}

\section{Donnerstag 18. April 1991}

18.30 Begrüssung durch Dr. Walter Graf

Präsident des Schweiz. Chemiker-Verbandes Begrüssung durch Dr. Francesco Brigatti Präsident der Associazione Ticinese Industrie Chimiche e Farmaceutiche (ATICEF)

Begrüssung durch Dr. F. Giovannini

Vertreter des Organisationskomitees

18.45 Vorstellung der Firma Helsinn SA, Biasca, durch Dr. Rolf Wandeler, Geschäftsleiter Helsinn Biasc

19.05 Vorstellung der Firma Inpharzam SA, Cadempino, durch Dr. Anibale Gazzaniga Geschäftsleiter Inpharzam Ricerche Taverne

19.25 Vorstellung der Firma Pharmaton SA, Bioggio. durch Dr. Costante Mombelli stv. Generaldirektor Pharmaton Bioggio

19.45 Vorstellung der Firma Sapec SA, Lugano, durch Dr. Attilio Melera, Geschäftsleiter Sapec Barbengo

20.00 Cocktail und gemeinsames Nachtessen im Hotel La Perla in gemütlichem Rahmen.

Übernachten im Hotel La Perla, Agno

Freitag, 19. April 1991

8.30- Besichtigung in Gruppen der Firmen Inpharzam SA

12.00 Pharmaton SA, Sapec SA

12.30 Mittagessen

14.15 72. Generalversammlung des Schweizerischen ChemikerVerbandes im Hotel La Perla, Agno

ca. 16.00 Ende der Versammlung

ca. 17.00 Besichtigung der Firma Helsinn SA, Biasca (Autobahn-Anschluss Biasca, Richtung Industrie-Quartier)

Die genaue Zeit wird am Ende der GV bekannt gegeben. 
nieurschule Winterthur, für seine Diplomarbeit: 'Kunststoff-Werkstoffe: Datenbanken, Verarbeitung, Charakterisierung'.

Dank

Dieses Jahr gilt mein besonderer Dank meinen Kollegen des Koordinationsausschusses. Die aktive und kompetente Mitarbeit der Herren W. v. Philipsborn, A. Merbach und $G$. Haas machten es möglich, dass wir bis Ende 1990 die entscheidenden Weichenstellungen zur Neugründung einer vereinigten Nachfolgegesellschaft für den Verband und die Gesellschaft machen konnten.

\section{Ausblick}

Am 18./19. April 1991 findet die 72. Generalversammlung und die Frühjahrstagung in Agno/Lugano im Tessin statt. Die Frühjahrstagung ist der Tessiner Pharmaindustrie gewidmet.

Im Herbst 1991 wird eine a.o. Generalversammlung, wahrscheinlich in Bern, stattfinden. Als einziges Traktandum wird die Auflösung des Chemiker-Verbandes und der Beitritt zur vereinigten Nachfolgegesellschaft zur Diskussion stehen.

\section{Walter Gra}

(Präsident)

Visp, Ende Februar 1991

\section{Schweizerische Chemische Gesellschaft} Sektion für Medizinische Chemie

Die Sektion für Medizinische Chemie (SMC) der Schweizerischen Chemischen Gesellschaft (SCG) wird am 22. Mai 1991 um 15.30 Uhr ihre zweite Mitgliederversammlung abhalten. Die Veranstaltung findet im Auditorium 510 bei Sandoz Pharma AG (Porte 5I, Hüningerstrasse 110, Basel) statt.

Auf dem Programm stehen u.a. eine Orientierung von Prof. W. von Philipshorn über den Stand und die Vorgeschichte des bevorstehenden Zusammenschlusses der SCG mit dem Schweizerischen Chemiker-

Verband (SChV), sowie zwei wissenschaftliche Vorträge von Prof. $H$. Timmermann (Amsterdam) 'Searching for selective ligands of histamine receptors'und Prof. Krogsgaard-Larsen (Kopenhagen) 'Drug Design Strategies in Alzheimer's Disease. Focus on Glutamic Acid, GABA and Acetylcholine'.

Interessenten sind herzlich eingeladen. Anmeldeformulare und weitere Auskünfte sind erhältlich beim SMC-Präsident Dr. E. Kyburz. c/o F. Hoffmann-La Roche $A G, 4002$ Basel.

\section{Neues Roche-Herz-Kreislauf-Medikament künftig gemeinsam von Roche und Marion Merrell Dow}

Marion Merrell Dow Inc. in Kansas City, Missouri/USA. und F.Hoffmann-La Roche AG in Basel, Schweiz. zusammen mit Hoffmann La-Roche Inc. In Nutley, New Jersey, haben soeben einen Vertrag unterzeichnet, der die gemeinsame Entwicklung und weltweite Vermarktung von Ro 40-5967, einem neuen Herzkreislauf-Medikament. vorsieht. Ro 40-5967 wurde in den Basler Laboratorien von Roche synthetisiert und stelt einen neuartigen Calciumantagonisten der dritten Generation dar: er befindet sich in Phase II der klinischen Entwicklung bei Bluthochdruck und Angina pectoris.

Fred W. Lyons, Jr., Präsident von Marion Merrell Dow Inc. kommentierte die Vereinbarung mit den Worten: „Wir meinen, dass unsere beiden Unternehmen sich ausgezeichnet in wissenschaftlicher Hinsicht wie auch im Marketing ergänzen und die notwendigen Ressourcen bereitstellen werden für die vollumfängliche Entwicklung und Vermarktung dieses vielversprechenden und für den Patienten äusserst wertvollen Medikaments. Wir erwarten eine lange und für beide Seiten lohnende Zusammenarbeit zwischen Marion Merrell Dow und Roche im Hinblick auf dieses Produkt."

Dr. Armin Kessler. Leiter der Pharma-Sparte und der konzernweiten operationellen Bereiche von F.Hoffmann-La Roche AG erklärte: „Normalerweise entwickeln und vermarkten grössere pharmazeutische Unternehmen ihre wichtigen, innovativen Substanzen selbst. Ro 40-5967 besitzt so interessante $\mathrm{Ei}$ genschaften. dass eine schnellstmögliche Entwicklung und Einführung am Markt erstrebenswert ist. Wir meinen, dass dies am besten durch die Kooperation unserer beiden Unternehmen erreicht werden kann, zumal sie sich in ihren Stärken vorteilhaft ergänzen."

Marion Merrell Dow vermarktet derzeit den Calciumantagonisten CARDIZEM (Diltiazem-HCl), ein gut eingeführtes Herz-KreislaufMedikament in den USA. Das Unternehmen bringt deshalb ein hohes Mass an Erfahrung in die gemeinsame Entwicklung und Vermarktung von Ro 40-5967 ein.
Schweizerischer Chemiker-Verband

Association Suisse des Chimistes

Schweizerische Chemische Gesellschaft

\section{Sehr geehrte Damen und Herren}

Wir freuen uns, unsere Mitglieder und Gäste zur Frühjahrstagung und zur ordentlichen Generalversammlung 1991 einzuladen.

Die Frühjahrstagung findet statt am

Donnerstag abend/Freitag, 18. und 19. April 1991

um 18.30 Uhr bzw. 08.30 Uhr, Hotel La Perla in Agno

The'ma der Tagung:

Aspekte der pharmazeutischen und chemischen Industrie im Tessin

Die Vorträge finden statt am Donnerstag, 18. April von 18.30-20.00 Uhr, Hotel La Perla, Agno. Besichtigt werden am Freitag, 19. April von 08.30-12.00 Uhr die Firmen Inpharzam, Pharmaton und Sapec. Die Firma Helsinn kann nach der GV am Freitag abend besichtigt werden.

Die Generalversammlung 1991 findet statt am

Freitag um 14.15 Uhr, Hotel La Perla, Agno

Die Frühjahrstagung wird gesponsort von den Firmen Helsinn SA, Biasca, Inpharzam SA, Cadempino und Taverne, Pharmaton SA, Bioggio und Sapec SA, Barbengo und von der Associazione Ticinese Industrie Chimiche e Farmaceutiche (ATICEF)

\section{Programm}

\section{Donnerstag 18. April 1991}

18.30 Begrüssung durch Dr. Walter Graf

Präsident des Schweiz. Chemiker-Verbandes Begrüssung durch Dr. Francesco Brigatti Präsident der Associazione Ticinese Industrie Chimiche e Farmaceutiche (ATICEF)

Begrüssung durch Dr. F. Giovannini

Vertreter des Organisationskomitees

18.45 Vorstellung der Firma Helsinn SA, Biasca, durch Dr. Rolf Wandeler, Geschäftsleiter Helsinn Biasc

19.05 Vorstellung der Firma Inpharzam SA, Cadempino, durch Dr. Anibale Gazzaniga Geschäftsleiter Inpharzam Ricerche Taverne

19.25 Vorstellung der Firma Pharmaton SA, Bioggio. durch Dr. Costante Mombelli stv. Generaldirektor Pharmaton Bioggio

19.45 Vorstellung der Firma Sapec SA, Lugano, durch Dr. Attilio Melera, Geschäftsleiter Sapec Barbengo

20.00 Cocktail und gemeinsames Nachtessen im Hotel La Perla in gemütlichem Rahmen.

Übernachten im Hotel La Perla, Agno

Freitag, 19. April 1991

8.30- Besichtigung in Gruppen der Firmen Inpharzam SA

12.00 Pharmaton SA, Sapec SA

12.30 Mittagessen

14.15 72. Generalversammlung des Schweizerischen ChemikerVerbandes im Hotel La Perla, Agno

ca. 16.00 Ende der Versammlung

ca. 17.00 Besichtigung der Firma Helsinn SA, Biasca (Autobahn-Anschluss Biasca, Richtung Industrie-Quartier)

Die genaue Zeit wird am Ende der GV bekannt gegeben. 


\section{Scientific Program HPLC 91}

Monday June 3

Plenary Session

9.00 Opening Ceremony

9.30 High Performance Capillary Electrophoresis

B.L. Karger, Northeastern University, Boston

10.15 Coffee break

Plenary Session

10.40 'High Performance Liquid Chromatography (HPLC),

Supercritical Fluid Chromatography (SFC), and Mass Spectrometry (MS) in Elucidation of Primary Structure of Glycoconjugate Glycans'

B. Fournet, Université des Sciences Techn., Lille

Parallel Session A: Electrokinetic Chromatography

11.30 'Factors Limiting Performance in Electrically Driven Capillary Separation Systems'

John H. Knox, University of Edinburgh, Edinburgh

11.50 'Electrochromatography'

Takao Tsuda, Nagoya Institute of Technology, Nagoya

12.10 'Initial Studies on the Use of Capillaries with Micron and Submicron Silica Particles in Electro-chromatography'

A. Najafi, B. Eray, R. K. Unger,

Johannes Gutenberg-Universität, Mainz

Parallel Session B: Preparative LC

11.30 'Comparison between Experimental and Calculated Band Profiles in Chromatography at High Concentrations'

G. Guiochon, University of Tennessee, Knoxville

11.50 'Process Scale HPLC. An Utopia or a Real Process Tool?' H. Colin, Separex S.A., Champigneulles

12.10 'Up-scaling and Calculation of Process Parameter in Production Preparative Chromatography from Analytical Data'

E. Eisenbeiss, E. Merck AG, Darmstadt

12.30 Lunch

13.30 Posters

Plenary Session

16.10 'HPLC : Quo Vadis?'

C.Horvath, Yale University, New Haven

17.00 Panel Discussion

HPLC vs. CZE and Electrochromatography

\section{Tuesday June 4}

Plenary Session 4

9.00 'Enantioselective Separations, State of the Art, Quo Vadis' W. Lindner, Universität Graz, Graz

9.45 'Enantioselective Chromatography in the Biomedical Sciences: New Frontiers for a Rapidly Developing Technique'

I.W. Wainer, McGill University, Montreal

10.30 Coffee break

Parallel Session A: Coupling Techniques

11.10 'GC-LC Coupling'

K.Grob, Kantonales Labor, Zürich

11.30 'Gradient Micro HPLC Coupled with SIMS for Drug Metabolism and Pharmaceutical Degradation Studies' Karin M. Kirkland, ICI Pharmaceuticals, Wilmington

11.50 'Sample Preatreatment at the Fmol/Amol Level Prior to GC-ECNI-MS for the Measurement of DNA Adducts' Roger U. Giese, Notheastern University, Boston

12.10 'Practical On-line HPLC Mass Determination Using Classical Light Scattering'

Gavin Dollinger, Bob Cunico, Michael Kunitani, Emeryville

Parallel Session B: New Phases

11.10 'Characterization of Adsorbent Surfaces in HPLC Using Isomeric Aromatic Hydrocarbons: Graphite, Alumina, Silica and Bonded Silicas'
Josef Kriz, Eva Adacova, John H. Knox,

Inst. of Chem. Technology, Prague

11.30 'Multi-legged Stationary Phases in Reversed-Phase Liquid Chromatography'

Kiyokatsu Jinno, Kunihiko Yamamoto, Tomoyuki

Kuwamoto, Takanori Ueda, Hideo Nagashima,

Kenji Itho, Toyohashi University of Technology, Toyohashi

11.50 'Mixed Layer Quaternary Ammonium Ion Exchange Adsorbents'

G. Pellaton, E.sz. Kovats, Ecole Polytechnique Federale de Lausanne, Lausanne

12.10 'Chromatography and Molecular Modelling'

Klara Valko, Peter Slegel, Hungarian Academy of Sciences, Budapest

12.30 Lunch

13.30 Posters

Parallel Session A: Enantioselective Separations

15.00 'Temperature Effects on the Separation of Enantiomers: A Critical Evaluation' Andreas A. Rizzi, University of Vienna, Vienna

15.20 Enantioselectivity at Extreme $\mathrm{pH}$ on a New Generation $\alpha \mathrm{l}$ Acid Glycoprotein Column'

Jörgen Hermansson, Norsborg

15.40 'Separation of Enantiomers Using Cellulase Silica as a Chiral Stationary Phase'

Curt Pettersson, Roland Isaksson, Göran Pettersson, Lennart Hansson, I. Marle, P. Erlandsson,

Biomedical Center, Uppsala

Parallel Session B: Supercritical Fluid Chromatography

15.00 'SFC as a Routine Chromatographic Technique in Pharmaceutical Industry'

K. Anton, M. Bach, M. Chalackal, Ciba-Geigy Ltd., Basel

15.20 'Extraction with Supercritical Fluids'

H. Engelhardt, J. Zapp, P. Haas, Universität des Saarlandes, Saarbrücken

15.40 'Studies of the Mechanism of Separation on Packed Columns in Supercritical Fluid Chromatography'

Roger H. Smith, Simon Cocks, Loughborough University of Technology, Loughborough, Leics

Plenary Session

16.10 'Supercritical Fluid Chromatography'

Karin Harkides, University of Uppsala, Uppsala

17.00 Panel Discussion SEC Quo Vadis?

\section{Wednesday June 5}

Plenary Session

9.00 'Mixed Mode Electrochromatography in Open Capillary Tubes'

Edward S. Yeung, William D. Pfeffer, Ames Laboratory, Ames

9.45 'Planar Chips Technology for Miniaturization and Integration of Separation Techniques Into Monitoring Systems' Andreas Hanz, J.C. Fettinger, E. Verpoorte, H. Lüdi, H.M. Widmer, Ciba-Geigy AG, Basel

10.30 Coffee break

Parallel Session A: Hicro LC

11.10 'Microcolumn LC: New Columns, Instrumentation and Applications'

Milos V. Novotny, Indiana University, Bloomington

11.30 'Trace Analysis in Capillary LC'

J. P. Chervet, M. Ursem, J. P. Salzmann, LC Packings, Amsterdam

11.50 'Use of RI Matching Fluids in a Laser Based RI Detector Suitable for Capillary Separations'

A. E. Bruno, B. H. Krattiger, F. Maystre, Ciba-Geigy Ltd., Basel

12.10 'Separation of Nitrogen and Oxygen Isotopes by Ionization Control Method. Optimization of Separation Conditions in HPCE and HPLC'

Nobuo Tanaka, Kazuhiro Kimata, Tetsuya Tanigawa, Ken Hosoya, Takeo Araki, Shigeru Terabe, Department of Polymer Sci., Kyoto 
Parallel Session B: Sample Preparation

11.10 'Sample Handling and Preparation Systems for Chromatography'

Udo Brinkman, Vrije Universiteit Amsterdam, Amsterdam

11.30 'Automated Sample Preparation for HPLC. Progress and Promises'

K.-Peter Hupe, Hewlett Packard, Waldbronn

11.50 'Direct Injection of Biological Fluids and Coupled-column LC Analysis of Marker Molecules'

K.-S. Boos, B. Wilmers, P. Marth, A. Walfort, J. Lintelmann, Klinikum Grosshadern University, München

12.10 'Enhancement of Protein Detection by Microwave-Induced Hydrolysis and OPA Derivatization'

M. Krämer, H. Engelhardt, Universität des Saarlandes, Saarbrücken

12.30 Lunch

13.30 Posters

Plenary Session

16.10 'Capillary HPLC and Electrokinetic HPLC'

Hans Poppe, University of Amsterdam, Amsterdam

17.00 Panel Discussion

Micro Systems: Is small really beautifull

\section{Thursday June 6}

Plenary Session

9.00 'Expert Systems in Chromatography'

D.L. Massart, Vrije Universiteit Brussels, Brussels

9.45 'LC-MS, SFC-MS and CZE-MS: The Current Status, Recent Developments and Future Dimensions'

D. E. Games, University College of Swansea, Swansea

10.30 Coffee break / Posters

Parallel Session A: Chemometrical Hethods

11.10 'HPLC Method Development Based on Multi-parameter Mapping'

L. R. Snyder, J, W. Dolan, D. C. Lommen, W. D. Raddatz,

Lafayette

11.30 'The Effect of Signal Noise on the Precision of

Chromatographic Data'

Eli Grushka, Arie Wanger, The Hebrew University,

Jerusalem

11.50 'Chemometric Methods Based on pH-induced Spectral Changes for Enhanced Peak Purity Assessment of Phenolic Solutes in LC'

A. F. Fell, J. B. Castledine, R. Modin, B. Sellberg, University of Bradford, Bradford

12.10 'Description of $\mathrm{pH}$ in HPLC'

Peter J. Schoenmakers, Rui M. Lopes Marques, Philips, Eindhoven

Parallel Session B: Biotechnology

11.10 'Identification by High Performance Displacement Chromatography (HPDC) of an Error in Translation of an Isoleucine Codon in $E$. coli'

John Frenz, Genentech Inc., South San Francisco

11.30 'Probing the Aggregation Phenomena of Recombinant DNA Derived Protein by a New Analytical Technique: Low Angel Laser Light Scattering Coupled On-line with Photodiode Array and Fluorescence Detector'

Shiaw-lin Uu, Jerry Cacia, William S. Hancock, Genentech Inc., South San Francisco

11.50 'High Performance Membrane Chromatography: An Alternative Method to the Preparative HPLC in Biotechnology'

Tatiana B. Tennikova, Frantisek Svec, Academy of Sciences of the USSR, Leningrad

12.10 'New Affinity Media for Large Scale Chromatography' Ken Jones, Freeport, Ballasalla, British Isles

12.30 Lunch

13.30 Posters
Plenary Session

15.00 'Preparative Supercritical Fluid Chromatography: Technological Developments and Applications' M. Perrut, Separex SA, Champigneulles

Parallel Session A: Polymer Characterisation

16.00 'Characterization of Macromolecules and Colloids by Asymmetrical-channel Flow Fjeld Flow Fractionation with Force-field Programming'

J. J. Kirkland, C. H. Dilks, Jr., and W. W. Yau, Du Pont, Wilmington, Delaware

16.20 'Size Exclusion Chromatography:Is it Really a Highperformance Technique'

Benny S. Welinder, Gentofte

16.40 'Observation Concerning Efficiency and Retention with Respect to Wall Effects and Fiber Porosity in Hollow Fiber Flow-FFF System (HFS)' Alf Carlshaf, Jan Ake Jönsson, University of Lund, Lund

Parallel Session B: Capillary Zone Electrophoresis

16.00 'Production, Characterization and Application of Polyacrylamid Gel Filled Capillaries for Electrophoresis G. Schomburg, J. A. Lux, H.-F. Yin, Max-Plank-Institut für Kohlenforschung, Mühlheim-Ruhr

16.20 'Tandem Capillary Enzyme Reactor-Capillary Zone Electrophoresis of Biological Substances' Wassim Nashabeh, Ziad El Rassi, Oklahoma State University, Stillwater,

16.40 'Application of Capillary Zone Electrophoresis for the Measurement of Drug-protein Binding' H. Poppe, J. Luijt, J. C. Kraak, University of Amsterdam, Amsterdam

\section{Friday June 7}

Plenary Session

9.00 'HPLC in Biotechnology in the 1990s'

Phyllis R. Brown, University of Rhode Island, Kingston

9.30 'Studies on Protein Conformation by High Performance Liquid Chromatography

A. Purcell, M. I. Aguilar, M. Wilce, A. Round, M. T. U.

Hearn, Monash University, Clayton, Victoria

10.00 'New Biomimetic Stationary Phases for Reversed Phase HPLC'

E. Bayer, Institut für Organische Chemie, Tübingen 1

10.30 Coffee break

11.00 'Future Applications of HPLC in Biotechnology'

William S. Hancock, Genentech, Inc., South San Francisco

11.45 'Antibodies as Tools in Separation Science'

Pred E. Regnier, Purdue University, W. Lafayette

12.30 Invitation to HPLC 92

12.45 Closing of HPLC 91

Farewell Drink

Topics of Poster Sessions

- Separation Techniques in Biotechnology

- Preparative Separations

- Enantioselective Separations

- Supercritical Fluid Chromatography

- Capillary Electrophoresis

- Electrokinetic Chromatography

- Microbore and Capillary LC

- Coupling Techniques

- Sample Preparation and Derivatisation

- Chemometrical Applications

- New Stationary Phases

- Biomedical, Pharmaceutical and Clinical Applications

- Ionchromarography

- Food Analysis

- Chromatographic Theory, Fundamentals

- Detection

- Polymer Characterisation

- Trace Analysis

- Other Applications

Approx. 450 Posters will be on display for 2 full days

(Monday + Tuesday or Wednesday + Thursday) 


\section{Personalia}

\section{Geburtstage}

\section{Willy Parpan}

Prof. Dr. Ing. Chem., Oberengstringen, Mitglied des SChV, feier am 2.4.91 seinen 65. Geburtstag.

\section{Hans Stettler}

Chemiker HTL, Basel, Mitglied des SChV, feiert am 2.4.91 seinen 70 . Geburtstag.

Hans Ulrich Theiler

Chemiker HTL, Ostermundigen, Mitglied des SChV, feiert am 3.4.91 seinen 65 . Geburtstag.

\section{Emil Beyeler}

ChemikerHTL, Wettingen, Mitglied des SChV, feiert am 9.4.91 seinen 70. Geburtstag.
Christian Klixbüll Jörgensen

Prof. Dr. phil., Geneve, Mitglied des SChV, feiert am 18.4.91 seinen 60. Geburtstag.

\section{Christoph Zinsstag}

Dr. phil. II, Mollens, Mitglied des SChV, feiert am 22.4.91 seinen 70 . Geburtstag.

Kurt M. Oesterle

Dr. Ing. Chem., Küsnacht, Mitglied des SChV, feiert am 30.4.91 seinen 90. Geburtstag.

\section{Neue Mitglieder}

Hans Michael Bürger

Dipl. Chem. ETH,

Lettenholzstrasse 15, 8038 Zürich

Hans-Rudolf Dettwiler

Dr. sc. techn.,

Hengart 15, 3902 Brig-Glis

Agatha Victoria Mugescu

Dipl. Ing. Chem.

Schulstrasse 29,5417 U. Sigenthal

Peter Wipf

Dr. phil. II

6315 5th Ave. No. 103,

Pittsburgh, PA 15206, USA

Samuel Wunderli

Dr. sc nat. ETH

Badgasse 8, 8402 Winterthur

\section{Société Vaudoise}

des Sciences Naturelles

Mercredi à $17 \mathrm{~h} 15$, Auditoire C, Collège Propedeutique Université de Lausanne, Dorigny

10 avril Prof. Dr. S. Leutwyler 24 avril „Chemicals reactions Inst. für Anorg. Anal. in isolated clusters" + Phys. Chemie Dr Hubert Mimoun, Universität Bern Firmenich S.A.

\section{Gratis-Unterlagen

\section{SChV Schweizerischer Chemiker-Verband}

Bitte um unverbindliche Unterlagen für die Mitgliedschaft

\section{CHIMIA}

kostenlose Probenummer, mit CHIMIA-Prospekt

Jahresabonnement für 1991

Schweiz: Fr. 156.- inkl. Porto

Ausland: Fr. 170.- inkl. Porto $\square$ Luftpostzuschlag: Fr. 69.-

Einzelnummer

Schweiz: Fr. 20.- inkl. Porto

Ausland: Fr. 25.- inkl. Porto

Einbanddecken

in Plastic, mit Halterung:

in Leinen (1990), ohne Halterung:

Fr. 21.- inkl. Porto $\mathrm{CH}$

Fr. 32.- inkl. Porto $\mathrm{CH}$

Jahreseinbände

$\square$ in Leinen, Inhalt angeliefert: Fr. 93.- inkl. Porto CH

\section{Frühere Jahrgänge} ungebunden

1988: Fr. 183.-inkl. Porto $\mathrm{CH}$

1989: Fr. 183.- inkl. Porto CH

1990: Fr. 183.- Porto CH gebunden

$\square$ Fr. 276.- inkl. Porto $\mathrm{CH}$

$\square$ Fr. 276.-inkl. Porto $\mathrm{CH}$

Fr. 276.- inkl. Porto $\mathrm{CH}$

Name

Firma

Strasse

Kennen Sie die Vorteile als Mitglied beim

Schweizerischen Chemiker-Verband?

Fordern Sie unverbindliche Unterlagen zur Information an.

Probenummern, Jahresabonnements, Einzelnummern, Einbanddecken, Jahreseinbände und frühere Jahrgänge der renommierten Fachzeitschrift CHIMIA können Sie mit nebenstehendem Talon bestellen.

Talon ausfüllen und an untenstehende Adresse senden:

CHIMIA-Abodienst

Postfach 124

CH-4010 Basel

Telefon (061) 7116060

Fax (061) 7119226 


\section{Personalia}

\section{Geburtstage}

\section{Willy Parpan}

Prof. Dr. Ing. Chem., Oberengstringen, Mitglied des SChV, feier am 2.4.91 seinen 65. Geburtstag.

\section{Hans Stettler}

Chemiker HTL, Basel, Mitglied des SChV, feiert am 2.4.91 seinen 70 . Geburtstag.

Hans Ulrich Theiler

Chemiker HTL, Ostermundigen, Mitglied des SChV, feiert am 3.4.91 seinen 65 . Geburtstag.

\section{Emil Beyeler}

ChemikerHTL, Wettingen, Mitglied des SChV, feiert am 9.4.91 seinen 70. Geburtstag.
Christian Klixbüll Jörgensen

Prof. Dr. phil., Geneve, Mitglied des SChV, feiert am 18.4.91 seinen 60. Geburtstag.

\section{Christoph Zinsstag}

Dr. phil. II, Mollens, Mitglied des SChV, feiert am 22.4.91 seinen 70 . Geburtstag.

Kurt M. Oesterle

Dr. Ing. Chem., Küsnacht, Mitglied des SChV, feiert am 30.4.91 seinen 90. Geburtstag.

\section{Neue Mitglieder}

Hans Michael Bürger

Dipl. Chem. ETH,

Lettenholzstrasse 15, 8038 Zürich

Hans-Rudolf Dettwiler

Dr. sc. techn.,

Hengart 15, 3902 Brig-Glis

Agatha Victoria Mugescu

Dipl. Ing. Chem.

Schulstrasse 29,5417 U. Sigenthal

Peter Wipf

Dr. phil. II

6315 5th Ave. No. 103,

Pittsburgh, PA 15206, USA

Samuel Wunderli

Dr. sc nat. ETH

Badgasse 8, 8402 Winterthur

\section{Société Vaudoise}

des Sciences Naturelles

Mercredi à $17 \mathrm{~h} 15$, Auditoire C, Collège Propedeutique Université de Lausanne, Dorigny

10 avril Prof. Dr. S. Leutwyler 24 avril „Chemicals reactions Inst. für Anorg. Anal. in isolated clusters" + Phys. Chemie Dr Hubert Mimoun, Universität Bern Firmenich S.A.

\section{Gratis-Unterlagen

\section{SChV Schweizerischer Chemiker-Verband}

Bitte um unverbindliche Unterlagen für die Mitgliedschaft

\section{CHIMIA}

kostenlose Probenummer, mit CHIMIA-Prospekt

Jahresabonnement für 1991

Schweiz: Fr. 156.- inkl. Porto

Ausland: Fr. 170.- inkl. Porto $\square$ Luftpostzuschlag: Fr. 69.-

Einzelnummer

Schweiz: Fr. 20.- inkl. Porto

Ausland: Fr. 25.- inkl. Porto

Einbanddecken

in Plastic, mit Halterung:

in Leinen (1990), ohne Halterung:

Fr. 21.- inkl. Porto $\mathrm{CH}$

Fr. 32.- inkl. Porto $\mathrm{CH}$

Jahreseinbände

$\square$ in Leinen, Inhalt angeliefert: Fr. 93.- inkl. Porto CH

\section{Frühere Jahrgänge} ungebunden

1988: Fr. 183.-inkl. Porto $\mathrm{CH}$

1989: Fr. 183.- inkl. Porto CH

1990: Fr. 183.- Porto CH gebunden

$\square$ Fr. 276.- inkl. Porto $\mathrm{CH}$

$\square$ Fr. 276.-inkl. Porto $\mathrm{CH}$

Fr. 276.- inkl. Porto $\mathrm{CH}$

Name

Firma

Strasse

Kennen Sie die Vorteile als Mitglied beim

Schweizerischen Chemiker-Verband?

Fordern Sie unverbindliche Unterlagen zur Information an.

Probenummern, Jahresabonnements, Einzelnummern, Einbanddecken, Jahreseinbände und frühere Jahrgänge der renommierten Fachzeitschrift CHIMIA können Sie mit nebenstehendem Talon bestellen.

Talon ausfüllen und an untenstehende Adresse senden:

CHIMIA-Abodienst

Postfach 124

CH-4010 Basel

Telefon (061) 7116060

Fax (061) 7119226 